\title{
Immunogenicity of the inactivated influenza vaccine in children who have undergone allogeneic haematopoietic stem cell transplant
}

\author{
Anne L. Ryan ${ }^{1}$ - Ushma D. Wadia ${ }^{2}$ Peter Jacoby ${ }^{3} \cdot$ Laurence C. Cheung $\mathbb{D}^{3,4} \cdot$ Fiona Kerr $^{1} \cdot$ Chris Fraser $^{5}$. \\ Heather Tapp ${ }^{6}$. Francoise Mechinaud ${ }^{7} \cdot$ Louise A. Carolan $^{8} \cdot$ Karen L. Laurie $^{8} \cdot$ Ian G. Barr $^{8}$. \\ Christopher C. Blyth ${ }^{2,9,10,11}$ - Nicholas G. Gottardo ${ }^{1,3,9} \cdot$ Peter C. Richmond ${ }^{2,9,10} \cdot$ Rishi S. Kotecha (iD) ${ }^{1,3,4,9}$
}

Received: 14 June 2019 / Revised: 14 October 2019 / Accepted: 15 October 2019 / Published online: 28 October 2019

(c) The Author(s), under exclusive licence to Springer Nature Limited 2019

\begin{abstract}
Influenza vaccination is recommended for children following allogeneic haematopoietic stem cell transplant (HSCT), however there is limited evidence regarding its benefit. A prospective multicentre study was conducted to evaluate the immunogenicity of the inactivated influenza vaccine in children who have undergone HSCT compared with healthy agematched controls. Participants were vaccinated between 2013 and 2016 according to Australian guidelines. Influenzaspecific hemagglutinin inhibition antibody titres were performed prior to each vaccination and 4 weeks following the final vaccination. A nasopharyngeal aspirate for influenza was performed on participants that developed influenza-like illness. There were 86 children recruited; 43 who had undergone HSCT and 43 controls. For the HSCT group, seroprotection and seroconversion rates were $81.4 \%$ and $60.5 \%$ for $\mathrm{H} 3 \mathrm{~N} 2,41.9 \%$ and $32.6 \%$ for $\mathrm{H} 1 \mathrm{~N} 1$, and $44.2 \%$ and $39.5 \%$ for $\mathrm{B}$ strain respectively. There was a significant geometric mean fold increase to the H3N2 (GMFI 5.80, 95\% CI 3.68-9.14, $p<0.001$ ) and B (GMFI 3.44, 95\% CI 2.36-5.00, $p=0.048)$ strains. Serological response was superior in age-matched controls to all vaccine strains. There were no serious adverse events following vaccination. For children who underwent HSCT, incidence of laboratory-proven influenza infection was $2.3 \%$. Overall, this study provides evidence to support annual inactivated influenza vaccine administration to children following HSCT.
\end{abstract}

Supplementary information The online version of this article (https:// doi.org/10.1038/s41409-019-0728-5) contains supplementary material, which is available to authorized users.

Rishi S. Kotecha

rishi.kotecha@health.wa.gov.au

1 Department of Clinical Haematology, Oncology and Bone Marrow Transplantation, Perth Children's Hospital, Perth, WA, Australia

2 Department of Infectious Diseases, Perth Children's Hospital, Perth, WA, Australia

3 Telethon Kids Cancer Centre, Telethon Kids Institute, University of Western Australia, Perth, WA, Australia

4 School of Pharmacy and Biomedical Sciences, Curtin University, Perth, WA, Australia

5 Blood and Bone Marrow Transplant Program, Queensland Children's Hospital, Brisbane, QLD, Australia

6 Department of Clinical Haematology and Oncology, Women's and Children's Hospital, Adelaide, SA, Australia

\section{Introduction}

Influenza infection can result in significant morbidity and mortality following allogeneic haematopoietic stem cell transplantation (HSCT) [1-3]. In children, the incidence of influenza following allogeneic HSCT ranges between 0.9

7 Children's Cancer Centre, Royal Children's Hospital, Melbourne, VIC, Australia

8 WHO Collaborating Centre for Reference and Research on Influenza, Victorian Infectious Diseases Reference Laboratory at the Peter Doherty Institute for Infection and Immunity, Melbourne, VIC, Australia

9 Division of Paediatrics, School of Medicine, University of Western Australia, Perth, WA, Australia

10 Wesfarmers Centre of Vaccines and Infectious Diseases, Telethon Kids Institute, University of Western Australia, Perth, WA, Australia

11 PathWest Laboratory Medicine WA, Perth, WA, Australia 
and $9.9 \%$ [4-8]. Administration of one dose of the seasonal inactivated influenza vaccine is recommended for children commencing 6 months following HSCT. Children aged 6 months to 8 years who have never previously been vaccinated should receive two doses, 4 or more weeks apart $[9,10]$. However, these recommendations are based on adult studies [10], with a distinct paucity of paediatricspecific data assessing the immunogenicity of the inactivated influenza vaccine following HSCT [11]. Given the lack of evidence, we undertook a prospective multicentre study to evaluate the immunogenicity of the seasonal inactivated influenza vaccine in children who have undergone allogeneic HSCT compared with healthy matched controls, and to identify risk factors that predict vaccine response in this population.

\section{Methods}

\section{Patient selection}

Children between the ages of 6 months and 18 years who were $\geq 6$ months and $\leq 2$ years post allogeneic HSCT were eligible. Recruitment was undertaken during the Southern Hemisphere influenza seasons of 2013-2016 (March to September) from four tertiary paediatric haematology, oncology and bone marrow transplant units in Australia (Princess Margaret Hospital for Children, Perth; Queensland Children's Hospital, Brisbane; Women's and Children's Hospital, Adelaide; The Royal Children's Hospital, Melbourne). Healthy siblings were recruited as age-matched controls. Exclusion criteria included anaphylaxis to previous doses of any influenza vaccine, a history of egg anaphylaxis, receipt of intravenous immunoglobulin within the last 3 months, a history of Guillain-Barre syndrome or current medical condition that would be compromised by inclusion in the study. Informed consent was obtained from the parents of each child prior to recruitment.

\section{Study design}

Participants were vaccinated with inactivated influenza vaccine according to national Australian guidelines [12]. Children receiving influenza vaccine for the first time post allogeneic HSCT were given two doses of the vaccine 4 weeks apart, with one dose given to vaccine-experienced children. For healthy age-matched controls, children less than 10 years of age receiving influenza vaccine for the first time, were given two doses of the influenza vaccine 4 weeks apart; children less than 10 years of age who had previously received the influenza vaccine and children who were 10 or older were given a single dose of the vaccine. A $0.25 \mathrm{~mL}$ dose was administered to children less than 3 years of age and $0.5 \mathrm{~mL}$ to children older than 3 . The inactivated trivalent vaccine was given for the 2013-2015 seasons and the inactivated quadrivalent vaccine for the 2016 season. The specific strains included in each annual influenza vaccine were A/Victoria/362/2011 (H3N2), A/California/7/2009 (H1N1), B/Hubei-Wujiagang/158/2009 (B) for 2013; A/ Texas/50/2012 (H3N2), A/California/7/2009 (H1N1), B/ Massachusetts/2/2012 (B) for 2014; A/South Australia/55/ 2014 (H3N2), A/California/7/2009 (H1N1), B/Phuket/ $3073 / 2013$ (B) for 2015 ; and A/Hong Kong/480/2014 (H3N2), A/California/7/2009 (H1N1), B/Phuket/3073/2013 (B), B/Brisbane/60/2008 (B) for 2016.

Blood was taken prior to each vaccination and 4 weeks following the final vaccination to assess influenza-specific immune responses. Following collection, blood samples were centrifuged and sera stored at $-20^{\circ} \mathrm{C}$. At the end of each influenza season, the samples were sent to the World Health Organization (WHO) Collaborating Centre for Reference and Research on Influenza, Victorian Infectious Diseases Reference Laboratory where standardised hemagglutinin inhibition (HI) assays were performed to determine specific influenza antibody titres towards each virus in the vaccine [13]. Viruses used for $\mathrm{HI}$ analysis were egg propagated; specifically for A(H1N1)pdm NYMC-X-179A (A/ California/7/2009) was used for seasons 2013-2016. For A (H3N2) IVR-165 (A/Victoria/361/2011), NYMC X-223A (hy A/Texas/50/2012), IVR-175 (A/South Australia/55/ 2014) and NYMC X-263 (hy A/Hong Kong/4801/2014) were used for the 2013, 2014, 2015 and 2016 seasons, respectively. Split B/Yamagata viruses B/Hubei-Wujiagang/158/2009, NYMC BX-51B (B/Massachusetts/02/ 2012) and B/Phuket/3073/2013 were used for the 2013, 2014 and 2015 seasons, respectively. Split viruses B/ Yamagata (B/Phuket/3073/2013) and B/Victoria (B/Brisbane/60/2008) were both used for the 2016 season.

Susceptibility to a vaccine-like strain was defined as a prevaccination $\mathrm{HI}$ titre of $<40$. Seroprotection in an individual was defined as a post vaccination HI titre of $\geq 40$. Seroconversion was defined as either a fourfold increase in $\mathrm{HI}$ antibody titre if the prevaccination titre was $\geq 10$ or a rise in HI titre from $<10$ to $\geq 40$ following vaccination [14]. The percentage $(95 \%$ confidence interval $[\mathrm{CI}])$ of patients who individually met the criteria for seroprotection and seroconversion to each strain of the vaccine was calculated.

Criteria as established by the Committee for Proprietary Medicinal Products (CPMP) were used to determine whether the vaccine was considered effective as to elicit an overall immunogenic response [15]. According to these criteria the influenza vaccine is considered effective if it meets one or more of the following three criteria: seroprotection in $>70 \%$ of patients; seroconversion in $>40 \%$ of patients; or a geometric mean fold increase (GMFI) of $>2.5$. These criteria were used to calculate one-sided $p$ values in relation to the 
null hypothesis for overall seroprotection and seroconversion to each strain of the vaccine. GMFI was calculated for each strain as the geometric mean of the fold increase in antibody level after vaccination, with $\mathrm{CI}$ and one-sided $p$ values estimated using a log-normal approximation for the distribution of antibody levels pre- and post-vaccination and the CPMP defined threshold of GMFI $>2.5$.

Linear regression models, with change in log antibody level as the dependent variable, were used to assess the influence of clinically relevant predictors on vaccine response to each strain. The predictor variables included within the models were age at receipt of first vaccination, the number of vaccination doses received, time since transplant, and the absolute lymphocyte count at the time of first vaccination. Lower than normal limits for absolute lymphocyte counts according to age were defined as $1.7 \times$ $10^{9} / \mathrm{L}$ for children $<5$ years of age, $1.1 \times 10^{9} / \mathrm{L}$ for 5 to $\leq 10$ years, and $1.0 \times 10^{9} / \mathrm{L}$ for $\geq 10$ years [16].

All patients enroled on the study that developed influenza-like illness were instructed to present for clinical review. Influenza detection was performed on a nasopharyngeal aspirate using polymerase chain reaction. Influenzalike illness was defined as an elevated temperature $\left(\geq 37.5^{\circ} \mathrm{C}\right)$ or a clear history of fever (e.g. chills, rigors); the presence of at least one constitutional symptom from irritability, myalgia, headache, vomiting, diarrhoea or malaise; and the presence of at least one respiratory symptom from cough, sore throat or rhinorrhoea; with the onset of symptoms occurring greater than $72 \mathrm{~h}$ after vaccine administration. Clinical features of all children with laboratory-proven influenza infection were documented.

This study was approved by the Child and Adolescent Health Service Ethics Committee (Ethics Approval Number 1988/EP), with ethical approval granted at all sites under the National Mutual Acceptance agreement. It conforms to the provisions of the Declaration of Helsinki in 1995 (as revised in Tokyo, 2004) and the National Statement on Ethical Conduct in Human Research, Australian National Health and Medical Research Council. The study was registered on the Australian New Zealand Clinical Trials Registry (ACTRN12614000240640).

\section{Results}

There were 86 children enroled in the study; 43 children who had undergone allogeneic HSCT and 43 healthy controls. The groups were frequency matched according to age (mean age: 7.7 versus 8.2 years, $p=0.65$ ) and sex (females: $37.2 \%$ versus $41.9 \%, p=0.66)$ Participant characteristics are listed in Table 1, with individualised characteristics listed in Supplementary Table 1. The percentage of patients in the allogeneic HSCT group that were susceptible to each
Table 1 Patient demographics

\begin{tabular}{|c|c|c|}
\hline Characteristic & $\begin{array}{l}\text { Allogeneic HSCT } \\
(n=43)\end{array}$ & $\begin{array}{l}\text { Healthy controls } \\
(n=43)\end{array}$ \\
\hline \multicolumn{3}{|l|}{ Age at vaccination } \\
\hline 6 months to $<3$ years & 9 & 6 \\
\hline 3 to $<10$ years & 18 & 24 \\
\hline 10 to $<18$ years & 16 & 13 \\
\hline \multicolumn{3}{|l|}{ Number of vaccine doses } \\
\hline One dose & 11 & 23 \\
\hline Two doses & 32 & 20 \\
\hline \multicolumn{3}{|l|}{ Diagnosis } \\
\hline Malignant & 26 & \\
\hline Non-malignant & 17 & \\
\hline \multicolumn{3}{|l|}{ Donor type } \\
\hline Matched sibling & 12 & \\
\hline Matched unrelated & 28 & \\
\hline Haploidentical & 3 & \\
\hline \multicolumn{3}{|c|}{ Interval between allogeneic HSCT and influenza vaccine } \\
\hline 6 to $<12$ months & 32 & \\
\hline$\geq 12$ months & 11 & \\
\hline \multicolumn{3}{|c|}{ History of graft versus host disease } \\
\hline Yes & 23 & \\
\hline No & 20 & \\
\hline \multicolumn{3}{|c|}{ On immunosuppressive therapy at time of vaccination } \\
\hline Yes & 13 & \\
\hline No & 30 & \\
\hline
\end{tabular}

strain of the vaccine prior to the first dose was $65 \%$ to $\mathrm{H} 3 \mathrm{~N} 2,88 \%$ to H1N1 and $91 \%$ to the B strain. Susceptibility of the healthy age-matched controls was $37 \%$ to $\mathrm{H} 3 \mathrm{~N} 2$, $70 \%$ to H1N1 and $79 \%$ to the B strain. Seroprotection occurred in $81.4 \%$ to $\mathrm{H} 3 \mathrm{~N} 2,41.9 \%$ to $\mathrm{H} 1 \mathrm{~N} 1$ and $44.2 \%$ to B strain in children who received allogeneic HSCT compared with $97.7 \%, 81.4 \%$ and $69.8 \%$ for healthy agematched controls (Table 2). Seroconversion occurred in $60.5 \%$ for $\mathrm{H} 3 \mathrm{~N} 2,32.6 \%$ for $\mathrm{H} 1 \mathrm{~N} 1$ and $39.5 \%$ for B strain in children who received allogeneic HSCT, while $72.1 \%$, $65.1 \%$ and $58.1 \%$ of healthy age-matched controls seroconverted to the respective strains (Table 2). For children in the HSCT group who received two doses of the vaccine and seroconverted, the second dose of the vaccine was required for seroconversion to occur in $47 \%$ of patients to $\mathrm{H} 3 \mathrm{~N} 2,70 \%$ to $\mathrm{H} 1 \mathrm{~N} 1$ and $81 \%$ to B strain.

According to CPMP criteria, children who had undergone allogeneic HSCT demonstrated a significant response to the H3N2 (GMFI 5.80, 95\% CI 3.68-9.14, $p<0.001$ and seroconversion $60.5 \%, 95 \%$ CI $45.9-75.1, p=0.003)$ and B (GMFI 3.44, 95\% CI 2.36-5.00, $p=0.048$ ) strains (Table 2). Healthy age-matched controls demonstrated significant response to all vaccine strains: H3N2 (GMFI 8.96, 95\% CI 5.96-13.45, $p<0.001$; seroprotection $97.7 \%$, 95\% 
Table 2 Overall immunogenicity to inactivated influenza vaccine in children who have undergone allogeneic HSCT and age-matched controls

\begin{tabular}{|c|c|c|c|c|c|c|}
\hline Strain & GMFI $(95 \%$ CI $)$ & $P$ value $^{\mathrm{a}}$ & Seroprotection \% (95\% CI) & $P$ value $^{\mathrm{a}}$ & Seroconversion $\%(95 \% \mathrm{CI})$ & $P$ value $^{\mathrm{a}}$ \\
\hline \multicolumn{7}{|c|}{ Allogeneic HSCT $(n=43)$} \\
\hline H3N2 (A) & $5.80(3.68-9.14)$ & $<0.001$ & $81.4(69.8-93.0)$ & 0.051 & $60.5(45.9-75.1)$ & 0.003 \\
\hline H1N1 (A) & $3.75(2.20-6.40)$ & 0.069 & $41.9(27.1-56.6)$ & $>0.99$ & $32.6(18.6-46.6)$ & 0.84 \\
\hline $\mathrm{B}$ & $3.44(2.36-5.00)$ & 0.048 & $44.2(29.3-59.0)$ & $>0.99$ & $39.5(24.9-54.1)$ & 0.53 \\
\hline B (2016) & $1.46(0.79-2.71)$ & 0.96 & $44.4(12.0-76.9)$ & 0.95 & $33.3(2.5-64.1)$ & 0.66 \\
\hline \multicolumn{7}{|c|}{ Controls $(n=43)$} \\
\hline H3N2 (A) & $8.96(5.96-13.45)$ & $<0.001$ & 97.7 (93.2-99.9) & $<0.001$ & $72.1(58.7-85.5)$ & $<0.001$ \\
\hline H1N1 (A) & $6.49(4.40-9.56)$ & $<0.001$ & $81.4(69.8-93.0)$ & 0.051 & $65.1(50.9-79.4)$ & $<0.001$ \\
\hline B & $6.81(4.41-10.53)$ & $<0.001$ & $69.8(56.0-83.5)$ & 0.51 & $58.1(43.4-72.9)$ & 0.008 \\
\hline B (2016) & $5.88(2.24-15.41)$ & 0.041 & $66.7(35.9-97.5)$ & 0.59 & $44.4(12.0-76.9)$ & 0.39 \\
\hline
\end{tabular}

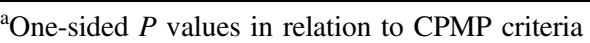

Table 3 Multivariate analysis of factors predicting GMFI to inactivated influenza vaccine in children who have undergone allogeneic HSCT

\begin{tabular}{|c|c|c|c|c|c|c|c|}
\hline \multirow[t]{2}{*}{ Variable } & \multirow[t]{2}{*}{ Influenza strain } & \multicolumn{2}{|l|}{ H3N2 (A) } & \multicolumn{2}{|l|}{ H1N1 (A) } & \multicolumn{2}{|l|}{ B } \\
\hline & & GMFI $(95 \%$ CI) & $P$ value & GMFI $(95 \%$ CI) & $P$ value & GMFI (95\% CI) & $P$ value \\
\hline \multirow[t]{2}{*}{ Time since transplant } & $<12$ months & $4.86(2.84-8.33)$ & \multirow[t]{2}{*}{0.42} & $2.65(1.44-4.87)$ & \multirow[t]{2}{*}{0.15} & $3.12(1.99-4.89)$ & \multirow[t]{2}{*}{0.24} \\
\hline & $\geq 12$ months & $9.66(3.85-24.24)$ & & $10.29(3.64-29.08)$ & & $4.54(2.11-9.76)$ & \\
\hline \multirow[t]{2}{*}{ Age at vaccination } & $<10$ years & $5.73(3.15-10.43)$ & \multirow[t]{2}{*}{0.97} & $5.31(2.69-10.48)$ & \multirow[t]{2}{*}{0.10} & $3.52(2.15-5.76)$ & \multirow[t]{2}{*}{0.58} \\
\hline & $\geq 10$ years & $5.91(2.71-12.86)$ & & $2.09(0.86-5.05)$ & & $3.30(1.74-6.27)$ & \\
\hline \multirow{2}{*}{$\begin{array}{l}\text { Lymphocyte count at time of } \\
\text { vaccination }\end{array}$} & Low & $3.17(1.15-8.76)$ & \multirow[t]{2}{*}{0.34} & $1.71(0.52-5.62)$ & \multirow[t]{2}{*}{0.30} & $1.59(0.71-3.57)$ & \multirow[t]{2}{*}{0.07} \\
\hline & Normal range & $6.80(4.03-11.46)$ & & $4.61(2.51-8.49)$ & & $4.22(2.78-6.39)$ & \\
\hline \multirow[t]{2}{*}{ Doses of vaccine received } & One & $7.05(2.77-17.98)$ & \multirow[t]{2}{*}{0.93} & $7.51(2.57-21.96)$ & \multirow[t]{2}{*}{0.54} & $1.88(0.90-3.94)$ & \multirow[t]{2}{*}{0.019} \\
\hline & Two & $5.42(3.13-9.38)$ & & $2.95(1.57-5.54)$ & & $4.23(2.74-6.53)$ & \\
\hline
\end{tabular}

GMFIs are raw subgroup means; $P$ values relate to a multivariate comparison of GMFIs between subgroup

CI 93.2-99.9, $p<0.001$ and seroconversion $72.1 \%, 95 \% \mathrm{CI}$ 58.7-85.5, $p<0.001$ ), H1N1 (GMFI 6.49, 95\% CI $4.40-9.56, \quad p<0.001$ and seroconversion $65.1 \%, 95 \%$ CI 50.9-79.4, $p<0.001$ ) and B (GMFI 6.81, 95\% CI $4.41-10.53, p<0.001$ and seroconversion $58.1 \%, 95 \%$ CI 43.4-72.9, $p=0.008$ ) according to CPMP criteria (Table 2).

The multivariate analysis of predictive variables revealed a significantly higher GMFI to the B strain for children who received two doses of the inactivated influenza vaccine (Two doses GMFI 4.23, 95\% CI 2.74-6.53 versus One dose GMFI 1.88, 95\% CI 0.90-3.94, $p=0.019)$ and who had a normal lymphocyte count at the time of vaccination (Normal range GMFI 4.22, 95\% CI 2.78-6.39 versus Low GMFI $1.59,95 \%$ CI $0.71-3.57, p=0.07$ ). Table 3 shows the multivariate analysis of factors predicting GMFI to each strain.

There were no adverse effects following vaccination in either the allogeneic HSCT or healthy age-matched control group. There was a single case of laboratory-proven influenza infection in the allogeneic HSCT group (2.3\%). This patient was typed as having H1N1pdm09 strain in 2014, with infection occurring 50 days following completion of a two dose immunisation schedule and absence of a serological response to any vaccine strain. The patient received a 5-day course of oseltamivir following confirmation of influenza infection and did not experience any significant influenza associated complications. There were no cases of laboratory confirmed influenza in the healthy age-matched control group.

\section{Discussion}

Influenza infection can result in significant morbidity and mortality following allogeneic HSCT [1-3]. Vaccination with inactivated influenza vaccine represents the main strategy to prevent infection. Several studies have been conducted to determine immunogenicity of the inactivated influenza vaccine in adults who have undergone HSCT, however, there is a distinct lack of paediatric-specific data [11]. Our study demonstrates that the inactivated influenza vaccine is safe and elicits a statistically significant serological response to $\mathrm{H} 3 \mathrm{~N} 2$ and $\mathrm{B}$ strains in children who 
have undergone allogeneic HSCT. However, serological response was limited in comparison with healthy agematched controls. The only study from which meaningful paediatric-specific data can be extrapolated for comparison between children who have undergone allogeneic HSCT is from a mixed paediatric-adult study [17]. In this study, seroconversion rates in 27 of the study participants under the age of 18 years were $59.3 \%$ for $\mathrm{H} 3 \mathrm{~N} 2,55.6 \%$ for $\mathrm{H} 1 \mathrm{~N} 1$ and $40.7 \%$ for B strain. Our current study shows comparable seroconversion rates to the $\mathrm{H} 3 \mathrm{~N} 2$ and $\mathrm{B}$ strains, but showed an inferior seroconversion rate to H1N1. There were notable differences between the H1N1 viruses tested in both of these studies as the Yalcin et al. [17] study was conducted over the 2007-2008 influenza season, when the previous seasonal influenza H1N1 vaccine was used, in comparison with our study, which used a post-2009 H1N1 pandemic influenza virus.

The CPMP have defined criteria to assess whether influenza vaccines are effective within a population. Using these criteria, our study shows that the inactivated influenza vaccine can be considered immunologically effective against the $\mathrm{H} 3 \mathrm{~N} 2$ and $\mathrm{B}$ strains in children following allogeneic HSCT. For the H1N1 strain, statistical significance was not achieved according to GMFI criteria and the numerical thresholds of $>70 \%$ for seroprotection and $>40 \%$ for seroconversion were not attained. However, interpretation of these findings should also take into consideration that the CPMP criteria were defined based on applicability to immunocompetent populations, further emphasised by satisfaction of the criteria against all three strains in our agematched healthy control population. This highlights the need for revised definitions according to different populations, such as immunocompromised children.

Previously, only two small studies assessing responses to influenza vaccination following HSCT have been exclusively performed in children [18, 19]. Haining et al. identified an increase in influenza A-specific CD4+ T-cell proliferation but no increase in influenza A-specific serum antibody levels in four children following vaccination with trivalent inactivated influenza vaccine 4-22 months following allogeneic HSCT [18]. Guerin-El Khourouj et al. identified a cellular proliferative response to $\mathrm{H} 1 \mathrm{~N} 1$ strain in 11 of 14 children following vaccination 76-336 days post allogeneic HSCT. H1N1 antibody titres were also measured in eight participants, with seroconversion only occurring in one patient following vaccination [19]. Although small numbers, both studies are important as they demonstrate that despite the absence of an influenza-specific antibody response, there was generation of an influenzaspecific T-cell response. This finding is supported by an adult study, which identified a strong cell-mediated immune response to a single dose of the trivalent inactivated influenza vaccine in 14 patients following allogeneic HSCT, despite a poor influenza-specific antibody response [20]. This further highlights the potential to consider alternative measures to determine vaccine efficacy in immunocompromised populations, in addition to those defined by the CPMP.

Receipt of two doses of the inactivated influenza vaccine and a normal lymphocyte count at the time of vaccination were identified as significant predictors of vaccine response to B strain. Two studies with combined adult and paediatric populations have identified time from transplantation to vaccination as one of the strongest predictors of response to trivalent inactivated influenza vaccine following HSCT $[21,22]$, with one of the studies also identifying absolute $\mathrm{CD} 19^{+}$cell counts as being predictive of response [22]. Although both studies were not able to identify a significant benefit for administration of two doses of the trivalent inactivated influenza vaccine compared with one dose $[21,22]$, the benefit of a second vaccine dose has been demonstrated in several studies which have assessed response to the AS03-adjuvanted $2009 \mathrm{H} 1 \mathrm{~N} 1$ vaccine in adults post HSCT [23-25], while another study identified comparable response to two doses of the AS03-adjuvanted $2009 \mathrm{H} 1 \mathrm{~N} 1$ vaccine in adults post allogeneic HSCT to a single dose received by healthy controls [26]. Transplant to vaccination interval $[24,26]$, presence of graft versus host disease [26], donor type [23] and receipt of immunosuppressive therapy [25] have all been shown to be predictive of response to the AS03-adjuvanted $2009 \mathrm{H} 1 \mathrm{~N} 1$ vaccine within these studies. Although this is the largest paediatric study to assess immunogenicity of the inactivated influenza vaccine following allogeneic HSCT, greater patient numbers are required to further validate the variables that have been assessed and to identify whether additional variables, such as presence of graft versus host disease, receipt of immunosuppressive therapy, donor type and stem cell source, predict response to vaccination.

To determine whether a vaccine is clinically effective traditionally relies on comparison of infection rate between unvaccinated and vaccinated subjects. In a previous retrospective study, the clinical efficacy of influenza vaccination in HSCT recipients was identified as $80 \%$ [27]. However, in the modern era, all patients who have undergone allogeneic HSCT are recommended to receive inactivated influenza vaccination [10], thus precluding assessment of vaccine efficacy due to the low number of unvaccinated patients. This highlights the need for alternative measures to determine the clinical efficacy of influenza vaccine for patients receiving HSCT. Incidence of laboratory-proven influenza infection was defined as the clinical endpoint for this study, limited to one case occurring in the HSCT cohort (2.3\%). This patient was typed as having H1N1pdm09 infection in 2014, occurring 50 days following completion of a two dose immunisation schedule, and did not mount protective 
antibody titres to any strain. The published data regarding clinical effectiveness of the influenza vaccine in HSCT recipients is limited, with reported incidence rates of $10.5 \%$ following one dose of the trivalent inactivated influenza vaccine and $4.6 \%$ following administration of two doses of AS03-adjuvanted $2009 \mathrm{H} 1 \mathrm{~N} 1$ vaccine post allogeneic HSCT [26, 27]. The low incidence of influenza infection in our study may also be influenced by minimising risk of exposure to infection, which is considered standard practice for children who have undergone HSCT [28].

In summary, our data demonstrate that the inactivated influenza vaccine is safe and provides immunogenicity to the $\mathrm{H} 3 \mathrm{~N} 2$ and $\mathrm{B}$ strains in children who have received allogeneic HSCT. We identify the need for further research to improve overall vaccine response and immunogenicity to the H1N1 pandemic strain. Receipt of two doses of the inactivated influenza vaccine and a normal lymphocyte count at the time of vaccination were both predictive of response to the B strain, however larger global collaborative studies are required to validate these findings and investigate a greater number of predictive variables. A high proportion of children who received two vaccine doses and seroconverted, required the second dose of the vaccine for seroconversion to each strain. In conclusion, our study provides evidence to support the recommendation for annual administration of inactivated influenza vaccine to children following allogeneic HSCT.

Funding RSK (NHMRC APP1142627) and CCB (NHMRC APP1111596) are supported by Fellowships from the National Health and Medical Research Council of Australia. This study was funded by a Perth Children's Hospital Foundation Project Grant and a Pfizer Cancer Competitive Research Grant. The WHO Collaborating Centre for Reference and Research on Influenza, Melbourne, is supported by the Australian Government Department of Health.

Author contributions UDW, PCR and RSK conceived and designed the study. ALR, FK CF, HT, FM and RSK recruited patients onto the study. LAC, KLL and IGB performed serological analysis. PJ performed statistical analysis. ALR, PJ and RSK analysed and interpreted the results. ALR and RSK wrote the paper. All authors edited and approved the final version of the paper for submission.

\section{Compliance with ethical standards}

Conflict of interest The authors declare that they have no conflict of interest.

Publisher's note Springer Nature remains neutral with regard to jurisdictional claims in published maps and institutional affiliations.

\section{References}

1. Ljungman P, Ward KN, Crooks BN, Parker A, Martino R, Shaw $\mathrm{PJ}$, et al. Respiratory virus infections after stem cell transplantation: a prospective study from the Infectious Diseases Working
Party of the European Group for Blood and Marrow Transplantation. Bone Marrow Transplant. 2001;28:479-84.

2. Nichols WG, Guthrie KA, Corey L, Boeckh M. Influenza infections after hematopoietic stem cell transplantation: risk factors, mortality, and the effect of antiviral therapy. Clin Infect Dis. 2004;39:1300-6.

3. Fisher BT, Danziger-Isakov L, Sweet LR, Munoz FM, Maron G, Tuomanen E, et al. A multicenter consortium to define the epidemiology and outcomes of inpatient respiratory viral infections in pediatric hematopoietic stem cell transplant recipients. J Pediatr Infect Dis Soc. 2018;7:275-82.

4. Styczynski J, Czyzewski K, Wysocki M, GryniewiczKwiatkowska O, Kolodziejczyk-Gietka A, Salamonowicz M, et al. Increased risk of infections and infection-related mortality in children undergoing haematopoietic stem cell transplantation compared to conventional anticancer therapy: a multicentre nationwide study. Clin Microbiol Infect. 2016;22:179.e1-10.

5. Hutspardol S, Essa M, Richardson S, Schechter T, Ali M, Krueger J, et al. Significant transplantation-related mortality from respiratory virus infections within the first one hundred days in children after hematopoietic stem cell transplantation. Biol Blood Marrow Transplant. 2015;21:1802-7.

6. Lujan-Zilbermann J, Benaim E, Tong X, Srivastava DK, Patrick CC, DeVincenzo JP. Respiratory virus infections in pediatric hematopoietic stem cell transplantation. Clin Infect Dis. 2001;33:962-8.

7. Srinivasan A, Wang C, Srivastava DK, Burnette K, Shenep JL, Leung W, et al. Timeline, epidemiology, and risk factors for bacterial, fungal, and viral infections in children and adolescents after allogeneic hematopoietic stem cell transplantation. Biol Blood Marrow Transplant. 2013;19:94-101.

8. Ryan AL, Kerr F, Gough H, Carter TL, Kotecha RS. Vaccinepreventable disease following allogeneic haematopoietic stem cell transplant in Western Australia. J Paediatr Child Health. 2019;55:343-8.

9. Rubin LG, Levin MJ, Ljungman P, Davies EG, Avery R, Tomblyn M, et al. 2013 IDSA clinical practice guideline for vaccination of the immunocompromised host. Clin Infect Dis. 2014:58:309-18.

10. Cordonnier C, Einarsdottir S, Cesaro S, Di Blasi R, Mikulska M, Rieger C, et al. Vaccination of haemopoietic stem cell transplant recipients: guidelines of the 2017 European Conference on Infections in Leukaemia (ECIL 7). Lancet Infect Dis. 2019;19:e200-12.

11. Dulek DE, de St Maurice A, Halasa NB. Vaccines in pediatric transplant recipients-past, present, and future. Pediatr Transplant. 2018;22:e13282.

12. Australian Technical Advisory Group on Immunisation. The Australian Immunisation Handbook, 10th Ed. Canberra: Australian Government Department of Health, 2013.

13. WHO Global Influenza Surveillance Network. Manual for the laboratory diagnosis and virological surveillance of influenza, 2011. Available from URL: https://www.who.int/influenza/gisrs_ laboratory/manual_diagnosis_surveillance_influenza/en/. Accessed 8th May 2019.

14. Fiore AE, Bridges CB, Katz JM, Cox NJ. Inactivated influenza vaccines. In: Plotkin S, Orenstein W, Offit PA (eds). Vaccines, 6th Ed. Philadelphia, PA: Elsevier 2013; pp 257-93.

15. Committee for Proprietary Medicinal Products (CPMP). Note for guidance on harmonisation of requirements for influenza vaccines. CPMP/BWP/214/96. European Agency for the Evaluation of Medicinal Products (EMEA). March 1997.

16. Comans-Bitter WM, de Groot R, van den Beemd R, Neijens HJ, Hop WC, Groeneveld K, et al. Immunophenotyping of blood lymphocytes in childhood. Reference values for lymphocyte subpopulations. J Pediatr. 1997;130:388-93. 
17. Yalcin SS, Kondolot M, Albayrak N, Altas AB, Karacan Y, Kuskonmaz B, et al. Serological response to influenza vaccine after hematopoetic stem cell transplantation. Ann Hematol. 2010;89:913-8.

18. Haining WN, Evans JW, Seth NP, Callaway GD, Wucherpfennig KW, Nadler LM, et al. Measuring T cell immunity to influenza vaccination in children after haemopoietic stem cell transplantation. Br J Haematol. 2004;127:322-5.

19. Guerin-El Khourouj V, Duchamp M, Krivine A, Pedron B, Ouachee-Chardin M, Yakouben K, et al. Cellular and humoral immunity elicited by influenza vaccines in pediatric hematopoieticstem cell transplantation. Hum Immunol. 2012;73:884-90.

20. Avetisyan G, Aschan J, Hassan M, Ljungman P. Evaluation of immune responses to seasonal influenza vaccination in healthy volunteers and in patients after stem cell transplantation. Transplantation. 2008;86:257-63.

21. Engelhard D, Nagler A, Hardan I, Morag A, Aker M, Baciu H, et al. Antibody response to a two-dose regimen of influenza vaccine in allogeneic $\mathrm{T}$ cell-depleted and autologous BMT recipients. Bone Marrow Transplant. 1993;11:1-5.

22. Karras NA, Weeres M, Sessions W, Xu X, Defor T, Young JA, et al. A randomized trial of one versus two doses of influenza vaccine after allogeneic transplantation. Biol Blood Marrow Transplant. 2013;19:109-16.

23. Engelhard D, Zakay-Rones Z, Shapira MY, Resnick I, Averbuch $\mathrm{D}$, Grisariu $\mathrm{S}$, et al. The humoral immune response of hematopoietic stem cell transplantation recipients to AS03adjuvanted A/California/7/2009 (H1N1)v-like virus vaccine during the 2009 pandemic. Vaccine. 2011;29:1777-82.

24. de Lavallade H, Garland P, Sekine T, Hoschler K, Marin D, Stringaris K, et al. Repeated vaccination is required to optimize seroprotection against $\mathrm{H} 1 \mathrm{~N} 1$ in the immunocompromised host. Haematologica. 2011;96:307-14.

25. Gueller S, Allwinn R, Mousset S, Martin H, Wieters I, Herrmann E, et al. Enhanced immune response after a second dose of an AS03-adjuvanted H1N1 influenza A vaccine in patients after hematopoietic stem cell transplantation. Biol Blood Marrow Transplant. 2011;17:1546-50.

26. Mohty B, Bel M, Vukicevic M, Nagy M, Levrat E, Meier S, et al. Graft-versus-host disease is the major determinant of humoral responses to the AS03-adjuvanted influenza A/09/H1N1 vaccine in allogeneic hematopoietic stem cell transplant recipients. Haematologica. 2011;96:896-904.

27. Machado CM, Cardoso MR, da Rocha IF, Boas LS, Dulley FL, Pannuti CS. The benefit of influenza vaccination after bone marrow transplantation. Bone Marrow Transplant. 2005;36: 897-900.

28. Tomblyn M, Chiller T, Einsele H, Gress R, Sepkowitz K, Storek J, et al. Guidelines for preventing infectious complications among hematopoietic cell transplantation recipients: a global perspective. Biol Blood Marrow Transplant. 2009;15: 1143-238. 